\title{
El Centro de Transferencia de Tecnología e Investi- gación Agroindustrial (CETTIA), de la Universidad Técnica Particular de Loja Ecuador
}

\author{
Ximena Carrión Granda* y Franco Jaramillo Montesinos **
}

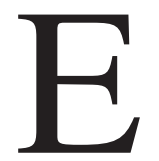

1 constante cambio tecnológico que caracteriza la economía actual, hace imperativa la actualización permanente de los recursos humanos de la empresa, la búsqueda y adaptación de tecnología idónea y el obtener y manejar la información necesaria para aprovechar oportunidades e innovar y, por lo tanto, competir de manera exitosa dentro de un sistema de apertura de mercados.

Sin embargo, el sector empresarial ecuatoriano, en su mayoría, sigue viendo a la innovación como un gasto y no como una inversión. La mayoría de las empresas en Ecuador no cuentan con investigación y desarrollo interno, y en muchos casos ni siquiera con departamentos técnicos efectivos que promuevan la generación de innovación endógena.

De esta manera puede afirmarse que la relación entre ciencia y desarrollo, así como la concepción de capital social de la investigación y del desarrollo científico y tecnológico, depende de la interacción entre educación e investigación. La primera como proceso de aprendizaje y la segunda como proceso de generación, adaptación y uso del conocimiento.

"La Universidad, al mismo tiempo que se transforma, debe preservar lo mejor de su tradición, que es la dedicación al saber y a la diversidad que cabe en lo universal de su misión, desde una perspectiva humana, con clave ética de lo que ha sido su dinamismo" (GARCÍA, 1997, p.33).

Las universidades representan una fuente cuantiosa de recursos de innovación en donde se concentra el personal humano con conoci-

* Directora del Centro de Investigación y Transferencia de Tecnología Agroindustrial de la Universidad Técnica Particular de Loja.

** Docente de la Universidad Católica de Bolivia, Sede Santa Cruz de la Sierra. 
mientos de alto valor agregado en áreas diversas, al igual que fuertes capacidades de investigación, desarrollo e innovación. Las universidades son, además, semilla para la generación de nuevas empresas innovadoras que surgen de los resultados comercializables de programas de investigación y desarrollo, del conocimiento que se acumula y de la formación de recursos humanos altamente capacitados. El carácter multidisciplinario de su propia organización, hace de la Universidad un excelente colaborador en el diseño e implementación de proyectos para la generación de una cultura tecnológica, de información, de integración económica, política y social, y de desarrollo sostenible.

Todo esto convierte a la Universidad en un socio deseable en el sector productivo para la promoción de la innovación. A pesar de ello, la brecha entre ambos sectores no ha disminuido a la velocidad necesaria en detrimento de ambos, siendo esto muy evidente en el caso de Ecuador. La Universidad se presenta como un generador de ciencia de alto nivel y tecnología de avanzada, sin embargo la falta de cultura tecnológica en las empresas ecuatorianas ha limitado la necesidad percibida de investigación, desarrollo e innovación que forma parte principal de la oferta universitaria.

En las respuestas que como institución propone a la sociedad la Universidad Técnica Particular de Loja, se pueden ver los esfuerzos de la universidad frente a los retos que le plantea su entorno y un nuevo planteamiento de investigación universitaria donde deja el modelo lineal de la misma para pasar a un modelo de investigación en paralelo. Esta institución, en su afán de promover el desarrollo de su entorno socioeconómico propone un modelo, donde recogiendo la esencia de los orígenes de las universidades, tiene como su ideal de vida "Desde El Humanismo Cristiano: Buscar la verdad y formar al hombre a través de la ciencia a fin de que sirva a la sociedad".

\section{La Universidad Técnica Particular de Loja y su nueva visión de uni- versidad}

La misión de la UTPL es: "Llevar a plenitud, desde la visión del humanismo cristiano, los ideales que dieron origen a las universidades: buscar la verdad y formar al hombre, a través de la ciencia, a fin de que sirva a la sociedad". Pretende, entonces, desde una formación integral que armonice la calidad profesional con los valores del humanismo cristiano, proyec- 
tarse en sus dimensiones, para que se constituya en foco de desarrollo económico, social, cultural, científico, humanístico, espiritual y ecológicamente sustentable de la región sur del Ecuador y de todo el país.

Las áreas de desarrollo estratégico de nuestra universidad son cinco, las mismas que reflejan nuestro esfuerzo y fortalezas frente a los retos del nuevo milenio:

* Tecnología de Punta en Computación.- Que es la tecnología del futuro y la indispensable para avanzar al desarrollo.

* Educación a Distancia.- Que es el "expertise" de la universidad, con la que hace posible llevar la educación superior a quienes quizá nunca podrían tenerla.

* Economía para el Desarrollo.- Aquí se une el potencial académico y la visión y horizontes de la Universidad para hacer posible que la misma sea parte de la sociedad a la que se debe.

* Recursos Naturales.- Siendo la mayor riqueza de nuestro país, la universidad busca la realización de una Gestión Ambiental acorde con el entorno, aprovechando y cuidando los recursos que poseemos.

* Humanidades.- Que busca potenciar la dimensión integral del hombre, imprescindible para la sociedad del siglo XXI.

Las funciones básicas de la Universidad: docencia, investigación y extensión, convergen hacia una "gestión productiva" que se plasma en realizaciones concretas para la sociedad en la que está inmersa. Esta "Gestión Productiva", hace posible el plasmar nuestras ideas en hechos concretos y mostrar al Ecuador y a Latinoamérica una nueva forma de hacer Universidad.

\section{La gestión productiva}

Se trata de una nueva forma de vida tanto institucional como personal. Pretende, principalmente, transformar al estudiante de un ser pasivo y eminentemente receptor, convertido en un simple espectador de los fenómenos sociales, en un ente dinámico y, por lo tanto, actor de primera línea en el proceso de romper el ostracismo en el que se encuentran atrapadas las universidades, las cuales "se van transformando, cada vez más, en urnas de cristal alejadas de la sociedad a la que se deben y en la que se encuen- 
tran, no siendo, en la mayoría de los casos, más que un elemento marginal en el desarrollo de los pueblos" (ROMERO, 1999, p.3).

La UTPL entonces con acciones concretas comienza a plasmar en hechos su visión, y para eso crea las denominadas "UNIDADES PRODUCTIVAS", que tomando las fortalezas que la Universidad posee en sus diversas áreas, se proyecta de manera eficaz a la sociedad. Siendo ejemplo de estas: Unidad de Proyectos y Sistemas Informáticos (UPSI), Centro de Apoyo y Desarrollo Empresarial y Social (CADES), Unidad de Diseño e Investigación en Arquitectura (UDIA), VIA Comunicaciones, entre otros.

Unidades que teniendo las características de la TRANSFERENCIA DE TECNOLOGÍA, sumada a la GESTIÓN PRODUCTIVA, dan como resultado un nuevo modelo de Universidad que plasma realmente su misión en hechos concretos dirigidos a la sociedad a la que se debe.

\section{La vinculación de la utpl con el sector agropecuario y agroindustrial}

Lamentablemente en nuestro país a causa de un inadecuado rol del Estado por la ausencia de competencia en ciertos subsectores, el heterogéneo y bajo nivel tecnológico, la falta de gestión de calidad y la debilidad empresarial, entre otras causas, uno de los mayores problemas que enfrenta la actividad agropecuaria, es la baja productividad.

Esto se manifiesta por el bajo grado de industrialización, el lento proceso de crecimiento de estos sectores, los bajos rendimientos de productos de consumo interno, en comparación con las explotaciones agrícolas y pecuarias de otros países similares, la poca mecanización de la superficie agrícola, etc., en los últimos 10 años, así como por el bajo valor agregado en relación al valor bruto de la producción, etc., entre otros indicadores, son las manifestaciones más agudas de esta baja productividad, que en el fondo no es más que una inadecuada aplicación de Ciencia y Tecnología en los sectores productivos.

La atención se enfoca entonces en una de las formas de lograr mejorar la productividad, que es la generación y transferencia de conocimientos hacia el sector agrícola e industrial, de manera que se haga investigación aplicada dirigida a la solución de sus problemas reales. El papel de las universidades en este contexto salta nuevamente a la vista como el eje dinamizador de este cambio, que por la velocidad que tienen los cambios, es cada vez más urgente. 
Una de las primeras facultades de la UTPL, es la de Ingeniería en Industrias Agropecuarias, que a través de sus laboratorios de Análisis Instrumental, Microbiología, Bromatología, Lácteos, Operaciones Unitarias, y Química, ofrecían sus servicios a los profesionales en formación y esporádicamente a empresas particulares, en especial a las de los sectores agroindustrial o alimenticio y minero.

A pesar de que estos laboratorios prestaban sus servicios a las empresas privadas, la relación con estas era totalmente esporádica, lo que sumado a las políticas, y a la forma de administración vigente en ese entonces, tanto para la realización de clases prácticas de laboratorio, como para servicios externos, no permitían un aprovechamiento eficiente de las instalaciones y recursos de los mismos, existiendo inclusive balances financieros negativos para la Universidad.

En resumen, las empresas del sector agroindustrial no se acercaban a la Universidad en busca de trabajos conjuntos, ya que la investigación académica no era atractiva ni permanente, y no se ajustaba por lo regular a las expectativas que estas empresas tienen.

\section{El centro de transferencia de tecnología e investigación agroindustrial (CETTIA)}

La implementación de las diferentes actividades de transferencia y vinculación requiere diversos servicios que permitan:

Regular adecuadamente las actividades a través de los contratos en los que se establezcan los derechos y obligaciones de cada parte, las condiciones de confidencialidad, la propiedad de los resultados y la explotación de los mismos.

Encontrar socios investigadores, tecnológicos, empresariales, financieros o usuarios más adecuados para el proyecto.

Acceder, mediante la preparación de propuestas, al mundo de la oferta de investigación científica a las empresas y demás instituciones que a través de las diversas administraciones apoyan las actividades de innovación tecnológica.

Proteger convenientemente los Derechos de Propiedad Intelectual e Industrial de modo que aporten ventajas competitivas a las organizaciones que los exploten económicamente. 
Facilitar todos los procesos administrativos ligados a las transferencias, posibilitando especialmente la disposición de recursos por parte de los investigadores.

La correcta gestión de los recursos económicos y humanos utilizados.

El CETTIA tiene como misión: Ser una unidad de interfaz del sistema Ciencia-Tecnología-Agroindustria, cuyo objetivo general es favorecer y enmarcar las relaciones entre el mundo de la investigación de la UTPL, y el de las empresas agroindustriales de su entorno socioeconómico, encauzando las necesidades y aprovechando las potencialidades y recursos respectivos, para convertirse en un verdadero centro de desarrollo científico.

De esta forma se enlaza a la Universidad con el sector productivo a través de investigación, desarrollo e innovación, transferencia de tecnología y asesoramiento, para la búsqueda de soluciones a los diversos problemas que se presentan en los sectores productivo, social y estatal afianzando la relación universidad-empresa-sociedad y fomentando el desarrollo sostenible de la región sur del país, que involucre al hombre y a la mujer como ejes centrales del beneficio de ese desarrollo.

Obviamente el CETTIA, es una unidad de servicios especializados que trabaja conjuntamente con otros CTT's de la Universidad, contribuyendo a plasmar y afianzar más aún el nuevo espíritu de la Universidad y su vinculación con la sociedad a la que se debe. El trabajo en equipo permite ampliar la gama de servicios a ofrecer por parte de la Universidad a las empresas agroindustriales: mercadeo, automatización de procesos, desarrollo administrativo y financiero, publicidad e imagen corporativa, entre otros.

La propuesta que el CETTIA hace al sector agropecuario y agroindustrial se plasma en lo que desde el Centro aportamos:

Mejoramiento de la rentabilidad empresarial.- $\mathrm{Al}$ ofrecer a las empresas un servicio de normalización, investigación, desarrollo de nuevos productos, certificación de calidad, investigación y mejoramiento de los procesos de producción, se pueden lograr una reducción considerable de costos en este tipo de rubros para estas empresas capaces o no de asumir por sí mismas los costos de una infraestructura necesaria para este tipo de labor, y por ende mejorar su eficiencia. 
Iniciativa y soluciones prácticas.- Nuestros laboratorios a través de 15 años de servicio, han conseguido una buena imagen a nivel local y nacional. Esto nos permite lograr con facilidad tener la confianza de las empresas para que requieran nuestros servicios y nos concedan la oportunidad de ofrecerles soluciones eficaces, rentables y prácticas en desarrollo de nuevos productos, mejoramiento de productos, innovación y mejoramiento de procesos, utilización de subproductos, entre otros.

\section{Discusión de resultados}

La discusión de resultados será enfocada desde la óptica de los instrumentos de vinculación de la Universidad con la empresa, los cuales se dividen en tres categorías de acuerdo a sus objetivos:

\section{a. Formalización y sistematización de la oferta de la Universidad}

* "La poca capacidad de investigación aplicada, que tienen las universidades latinoamericanas, y que en sí misma no tiene ninguna relevancia social, es uno de los problemas más graves de estas" (ARREDONDO, 1985, p.95). Desde la creación del CETTIA se han incorporado mecanismos que cambian esta realidad. Los trabajos de investigación y transferencia de tecnología hasta ahora realizados por el CETTIA, han dado resultados que nos permiten expresar claramente que la Universidad ha sido beneficiada no solo en el ámbito económico, sino también en la obtención de experiencia en este tipo de trabajos y proyectos. Fuera de la Universidad está claro que la búsqueda de investigación aplicada genera resultados inmediatos en la sociedad.

* Si bien los laboratorios de la Universidad habían ganado un prestigio determinado a nivel local, regional y nacional, también es cierto que debido al sistema administrativo con el que operaban se podía observar un estancamiento de su labor durante aproximadamente 5 años. Ahora los laboratorios han ampliado su gama de servicios y su prestigio ha incrementado considerablemente, pues la relación con empresas importantes del país está empezando a rendir frutos.

* Tanto las empresas como las universidades necesitan manejar un mismo lenguaje en los procesos de vinculación entre ellas. Para esto es necesario capacitación de los profesores, investigadores, y técnicos. 
El CETTIA desde su creación ha realizado un arduo trabajo en este campo buscando y motivando al personal que trabaja en sus diferentes áreas a mantener una permanente capacitación.

* Si se busca dar relevancia a los programas académicos y de investigación en las universidades, la acción realizada por el CETTIA, conjuntamente con las Facultades de Ingeniería en Industrias Agropecuarias e Ingeniería Química, al implementar el modelo de pasantías en reemplazo al antiguo sistema de prácticas, empieza a mostrar cómo esta labor permite que el profesional en formación adquiera destrezas y experiencia real dentro de su proceso de formación. Esto contrasta radicalmente con el antiguo sistema, donde el aprendizaje era repetitivo y el conocimiento y manejo de equipos era casi nulo.

\section{b. Impulso de la transferencia de resultados de I + D y la búsqueda de resultados aplicables}

Se ha analizado que por lo regular las universidades no han sido capaces de transferir la escasa investigación y tecnología aplicada, generada en su seno, debido a que no se ha tomado en cuenta que dicha tecnología debe ser adecuadamente adaptada a las necesidades de sus últimos usuarios. Así observamos cómo los distintos cursos, talleres, seminarios que se han realizado a través y/o con colaboración del Centro han dado resultados concretos de estas acciones. Ejemplo de esto es como uno de los primeros seminarios realizados por el Centro, "Cultivo en invernaderos, una alternativa para el nuevo milenio" ha impulsado efectivamente el interés por este sistema de cultivo en la ciudad y provincia, lo que se puede apreciar en el incremente del número de unidades de invernaderos en el sector.

La investigación en las universidades ha estado caracterizada por su poca o casi nula implicación social. El $70 \%$ de las tesis de grado de la Facultad de Ingeniería en Industrias Agropecuarias se refieren a proyectos de factibilidad que, según los resultados explícitos en los mismos, son proyectos muy atractivos y con buena rentabilidad. Sin embargo, ni siquiera el 1\% de estos han sido ejecutados. El CETTIA en colaboración directa con el decanato de esta facultad ha empezado a buscar mecanismos que permitan cambiar esta realidad. Así, ya se han propuesto temas de trabajos de investigación y tesis que son de necesidad de empresas, instituciones y la misma Universidad, convirtiendo este 
esfuerzo de profesores y estudiantes en la investigación que tiene pertinencia en el entorno socioeconómico de la Universidad.

\section{c. La búsqueda de acciones de conocimiento, comunicación y cola- boración entre las partes}

Una dificultad importante de la vinculación de las universidades con su entorno socioeconómico ha sido que sus sistemas administrativos no son flexibles en la realización de trabajos o proyectos de investigación con empresas o instituciones. Esto produce una obstaculización en el trabajo y la obtención de los resultados que es lo "único" que los clientes esperan de la Universidad. El CETTIA ha propuesto un modelo de administración en el cual la administración del Centro se encarga de agilitar los procesos derivados de este tipo de trabajos. Esto ha permitido, con algunas dificultades, que la obtención de resultados en los trabajos que nos han sido encomendados sean presentados en los plazos previstos. Así también, tanto el Centro como la Universidad pueden mantener un control sobre la contabilidad general de los trabajos y también que los directores de los mismos sean conscientes de la optimización de los recursos de la Universidad al momento de la ejecución de dichos trabajos.

Por mucho tiempo la UTPL estuvo aislada del contexto investigativo, científico y social. Las actividades tanto del CETTIA como de las demás unidades productivas de la Universidad han ido cambiando esta realidad. Los demás actores del entorno social y productivo (empresas, ONGs, otras universidades, etc.) miran a la UTPL como un socio potencial y atractivo para sus proyectos de I + D + I. Otro ejemplo de esto son los distintos consorcios, redes, alianzas, convenios, en los cuales la Universidad a través de sus distintas unidades productivas participa directamente.

\section{Ejemplo de acciones y resultados concretos}

\section{Desarrollo y mejoramiento competitividad empresarial:}

Se han realizado varias acciones a empresas que ha ayudado sin duda alguna al mejoramiento de rentabilidad global, pero vale la pena anotar en este punto el trabajo realizado a un empresario de la región 
quien contrató toda una asesoría y diseño de una nueva fábrica de bebidas a partir del jugo de caña. Desde la elaboración del estudio de mercado, diseño de líneas de proceso e investigación de la formulación del producto, se realizó con personal de la Universidad con la ayuda de estudiantes de diversas carreras; una vez montada la empresa (la que se encuentra en la fase final) se dará trabajo directo a 150 personas e indirecto a más de 600 .

Otro punto vital es que como centro de transferencia de tecnología se ha trabajado para lograr que nuestros laboratorios de análisis de alimentos sean calificados para otorgar Registro Sanitario, cuyo trámite se tenía que realizar en una de las dos ciudades más grandes del país y demoraba algo más de año y medio obtenerlo. Con nuestro laboratorio calificado no solo se ha eliminado el problema de la centralización y demora en el tiempo, si no que se han elaborado varios programas con el fin de mejorar la competitividad de las empresas de la región que permitirá que productos ecuatorianos se comercialicen en el norte del Perú.

A través de una alianza con la empresa privada se realizó un seminario taller sobre cultivo en invernaderos, esto día un gran impulso a esta actividad en la región, lo que se demuestra de los datos tomados del Ministerio de Agricultura, en los que se aprecia como luego de 8 meses de concluido el seminario, se instalaron en la ciudad y los alrededores 220 invernaderos, con una capacidad aproximada de producción de 200 toneladas de alimentos.

\section{Desarrollo comunitario}

Se han hecho varias acciones con comunidades rurales, sin embargo vale la pena mencionar la realizada con una comunidad de ganaderos en una zona rural de la provincia. Se dio capacitación a 150 pequeños ganaderos con la finalidad de mejorar la producción. Hecho el seguimiento respectivo y de los datos tomados sobre la producción, está aumento en un $167 \%$ a los seis meses de finalizada la asesoría.

Con una empresa de productos lácteos se realizó la implantación de un sistema de recolección de leche en una zona muy apartada de la zona oriental del país, con este sistema se permitió que los pequeños productores puedan vender su producción y recibir asesoría y capacitación en esta área. 


\section{La experiencia interna}

Ya se ha hablado de las dificultades de establecer relaciones entre la Universidad y la empresa así como la de ejecutar sistemas de innovación, sin embargo la experiencia obtenida en este espacio de tiempo ha sido muy enriquecedora. El modelo de trabajo implantado en donde estudiantes y profesores convergen juntos para trabajar en proyectos e investigaciones si bien es cierto no es nuevo en el mundo, pero en Latinoamérica es muy poco visto, sobre todo en nuestro país y en nuestras universidades. La UTPL ha logrado insertar esta nueva visión, primero al interno de la misma Universidad, que ha dado como resultado un proceso de innovación interno en donde continuamente se cambian y analizan los procesos, logrando que la administración se flexibilice, que decanos y directores apoyen la realización de trabajos de investigación y de tesis aplicadas a la realidad; todo esto sin los "traumas" naturales que se producen al realizar cambios en estructuras que hasta entonces habían sido demasiados rígidas.

La interdisciplinariedad ha visto en esta nueva forma de trabajo de la UTPL, un nicho apropiado para su expansión y crecimiento, cuando se realiza un proceso de adaptación y luego de transferencia de tecnología, en cualquier dirección, se establecen vínculos que permiten que la colaboración no termine al finalizar el trabajo, sino que sea el inicio de una nueva idea, o un nuevo proyecto.

El futuro de nuestras universidades latinoamericanas depende de la gestión productiva que permita financiarlas, más allá de aportes estatales y matrículas. Toda la Universidad debe ser una gran empresa colectiva capaz de gestar docencia, investigación y extensión en relación con la sociedad a la que se debe, promoviendo su desarrollo y a la vez obteniendo de ella a través de proyectos diversos, asesorías, servicios, investigaciones, etc., los fondos que le son necesarios en la cantidad suficiente. Es una ganancia para todos; hasta para los profesores, que empiezan a tener al fin una remuneración más allá de la subsistencia.

Hay otras muchas más dimensiones: Las repercusiones éticas de la actividad científica; la necesidad del diálogo interdisciplinario; el trabajo en equipo; la necesidad de planes flexibles tanto a corto y medio plazo, como a largo plazo, y la conjugación de ciencia aplicada con la básica, cuya aplicación final es más dilatada, pero imprescindible; el establecimiento de prioridades con la suficiente capacidad de modifica- 
ción según las circunstancias futuras como para que no sea una camisa de fuerza; los valores universales de la actividad investigadora; y un largo etcétera de ese "genio colegiado" que es la investigación en nuestras universidades que tanto necesita nuestra sociedad, no como lujo superfluo, sino como hipoteca de un futuro más humano y más justo.

\section{CONCLUSIONES}

La aplicación de una gestión tecnológica no puede ser inventada ni manejada de cualquier manera, es conveniente tener referentes que indiquen al menos por donde no se debe caminar, es así como el CETTIA en su concepción a recogido lo mejor de muchas propuestas en este campo, adaptándose a la realidad de su entorno, pero sin perder de vista los ideales ni la visión y misión de la Universidad a la que se pertenece. Si se quiere y se busca una efectiva vinculación de las universidades con el sector empresarial, un paso muy importante es la formación de "gestores" de vinculación, ya que estos son los encargados de traducir la visión empresarial a la Universidad y viceversa.

Se debe tener definidos los mecanismos y personas mediante los cuales se puede acceder a servicios de la Universidad. Los empresarios no tienen "tiempo" para buscar en la Universidad con quien dirigirse, lo deben tener claro desde antes del inicio de la vinculación.

El modelo de GESTIÓN PRODUCTIVA, ha mostrado su eficacia cambiando radicalmente el antiguo modelo universitario, pero esto debe continuar de manera que se busque además un conocimiento más fondo de la realidad empresarial con, por ejemplo, convenios con empresas para la realización de una Gestión Productiva en la misma empresa. El CETTIA, maneja un modelo administrativo innovador con respecto a los tradicionales, desde el momento en que la Universidad confía al mismo Centro la administración de las áreas neurálgicas del proceso de transferencia de tecnología. Sin embargo en este campo no se ha dicho la última palabra, ya que el constante cambio de la reglas de vinculación entre la Universidad y la empresa, hace que los modelos administrativos se encuentren en permanente cambio.

Se debe prestar mucha atención a los criterios básicos para el desarrollo de las habilidades de valoración, negociación y protección de los derechos de propiedad intelectual. Se han de establecer normas claras para el efecto que permitan la facilidad y motivación de las empre- 
sas para trabajar con las universidades; teniendo en cuenta que costeo, valoración, negociación y protección de la propiedad intelectual son temas complementarios entre sí, al momento de realizar trabajos conjuntos con empresas o instituciones de cualquier tipo.

Los sistemas de prácticas integrales, se convierten en una necesidad en los procesos de enseñanza universitaria. Es preciso formar profesionales no para un empleo, sino para procesos de creación de empresas. El CETTIA a través de su nuevo sistema de pasantías va por esta línea y busca continuamente su mejoramiento.

Se debe promover el fortalecimiento de redes entre universidades, que permitirá una oferta más sólida de servicios. El trabajo de cada Universidad por separado no se puede comparar con las acciones conjuntas. Una red nacional de CTT's puede ser un mecanismo que puede ser de gran ayuda en esta tarea.

Un gran problema de la educación en el Ecuador y en América Latina, es sin duda alguna, la poca capacidad de respuesta al momento de buscar soluciones a los problemas de la investigación, se debe empezar entonces a buscar formas de incorporar estos conceptos en la educación superior.

Para finalizar, el mecanismo de vinculación Universidad-sector productivo, más eficaz, son los Centros de Transferencia de Tecnología, el CETTIA es ejemplo de cómo tomando los conceptos fundamentales de la transferencia de tecnología y aplicándolos a una realidad concreta, sin olvidar el rol fundamental de las universidades, se puede realizar una vinculación efectiva entre la Universidad y su entorno.

Las tesis de grado deben convertirse en un lugar común de encuentro en las relaciones Universidad-empresa, mas no darle la razón a una de las famosas frases consigna de la Escuela de Negocios de Harvard, cuando dice que: "nunca dé una orden que usted sabe que no se va a cumplir". Existen muchas formas para solucionar esto. Una de ellas es darle más dosis de realidad a los profesores y vincularla al entorno socioeconómico para que presente propuestas específicas.

Las universidades manejan el concepto "tiempo" con diferentes prioridades que las empresas. Esto hace que se alejen más. La solución está en tener en las universidades el concepto claro, al empresario no le interesan los problemas "domésticos" de las mismas, solo buscan resultados, se puede tener por ejemplo un grupo de "contacto" que maneje perfectamente los dos lenguajes: el universitario y el empresarial. Sobre este grupo estriba el éxito de la relación. 


\section{REFERENCIAS}

1. Arredondo, Víctor M. "La educación superior y su relación con el sector productivo". México. Asociación Nacional de Universidades.

2. Calleja, T. "La Universidad como empresa: Una revolución pendiente”. España. Editorial Rialp.

3. García Guadilla C. "La juventud universitaria en América Latina". CRESALC/UNESCO-ILDIS. Venezuela

4. Romero, Luis M. et al. "Restauración de la Universidad en Latinoamérica: Desde los orígenes hacia una nueva visión”. Ecuador, III Encuentro de la Red de Universidades Andinas.

\section{BIBLIOGRAFÍA}

Agueda Rodríguez, Cruz. "La Universidad en la América Hispánica”. España. Editorial Mapfre.

Brunner, José J. "Cultura y Educación en el horizonte del año 2000", URSHLAC-UNESCO. Serie Estudios y Documentos No. 7. Venezuela.

Brunner, José J. "Educación Superior, Investigación Científica y Transformaciones Culturales en América Latina"; Vinculación Universidad - Sector Productivo, BID-CECAB-CINDA, Chile.

CEPAL, "Transformación Productiva con equidad. La tarea prioritaria del desarrollo de América Latina y el Caribe en los años noventa". Chile.

CEPAL/UNESCO, "Educación y Conocimiento: eje de la transformación productiva con equidad". Chile.

Clark Burton J., 1998. "Creando Universidades Emprendedoras". Revista Valenciana de Estudios autonómicos.

Conesa, F. "Las oficinas de Transferencia de Resultados de Investigación en el sistema español de innovación". Tesis doctoral dirigida por el Dr. I. Fernández de Lucio. Universidad Politécnica de Valencia. 
Días Rodríguez Marco, "Tendencias y retos en la educación superior". CRESALC-UNESCO. Venezuela.

Escardibul, B. et al. 1999. "Evaluación de departamentos de la Universidad de Lleida implicados en los acuerdos universidad-empresa en el período 1994 - 1998”. VIII Seminario Latino-Iberoamericano de Gestión Tecnológica. Revista Valenciana d'Estudis Autonómics.

Escardibul, B. et.al. 1997. "La transferencia de Tecnología en la Universitat de Lleida

(UdL). Evaluación de los resultados del Centro de Transferencia Tecnológica en el período 1994 - 1997”. Primer Seminario Nacional de Gestión Tecnológica ALTEC - AMTEC'98. Universidad Autónoma de Yucatán, en Mérida, México.

Esquivel Juan, "La Universidad. Hoy y mañana" México. AUNIES/UNAM.

Frame, J.D. "La dirección de proyectos en las organizaciones". España. Editorial Granica.

García Guadilla C. "La juventud universitaria en América Latina". Venezuela.

CRESALC/UNESCO-ILDIS,

García Guadilla C. "Conocimiento, Educación Superior y Sociedad en América Latina” CENDES. Venezuela. 1992

Garcia Guadilla C., 1993. "Expansión y diferenciación del sector privado de educación superior en América Latina”, Educación Superior, No. 26. CRESALC/UNESCO.

Levy Daniel, "La calidad en las universidades Latinoamericanas: vino viejo en odres nuevos". Documento preparado para la reunión de Rectores, BID Y UDUAL, Washington.

Johan Smith, "La Investigación en Latinoamérica “ Editora Banco Mundial. 
Muñoz Carlos, 1988. “Una conversación sobre la investigación educativa en la Universidad." Revista Universidad Futura. No. 6.

Pérez, A. et. al.

“Relaciones Universitat-empresa”. Departament d'Industria i Energía de la Generalitat de Catalunya i Universitat Politécnica de Catalunya.

Revista Económica de Catalunya. 